\title{
Let Graphics Tell the Story - Datasets in R
}

by Antony Unwin, Heike Hofmann and Dianne Cook

Abstract Graphics are good for showing the information in datasets and for complementing modelling. Sometimes graphics show information models miss, sometimes graphics help to make model results more understandable, and sometimes models show whether information from graphics has statistical support or not. It is the interplay of the two approaches that is valuable. Graphics could be used a lot more in $\mathrm{R}$ examples and we explore this idea with some datasets available in $\mathrm{R}$ packages.

\section{Introduction}

One of the attractive features of $\mathrm{R}$ and its package system is that it allows the use of real datasets in the examples and vignettes to demonstrate statistical analyses. While we usually associate $\mathrm{R}$ with statistical methodology, it is hard to underestimate its impact as a data archive and dissemination tool. R has long ago overtaken data archives such as StatLib (Vlachos and Meyer, 1989 -) or OzDASL (Smyth, 2011) in scope and users. There are over 3000 data objects included in the 1001 packages available on CRAN, that have been submitted just before or after the release of R 2.15.2 (Trick or Treat) on Oct 26 2012. Not all of the objects are datasets in the traditional sense, that we deal with a two dimensional object with variables in the columns and records in the rows, but we were able to extract information on the numbers of columns and rows for 2428 of them. Figure 1 gives a summary of the number of packages and the number of associated datasets. A table of all packages and associated data objects can be found at http://www. public. iastate.edu/ hofmann/data_in_r_sortable.html.

For some of these datasets, graphics are also included, though the emphasis is usually more on modelling and analysis. In this article we want to show the value of including more graphics in $\mathrm{R}$ examples and to illustrate how they complement modelling.

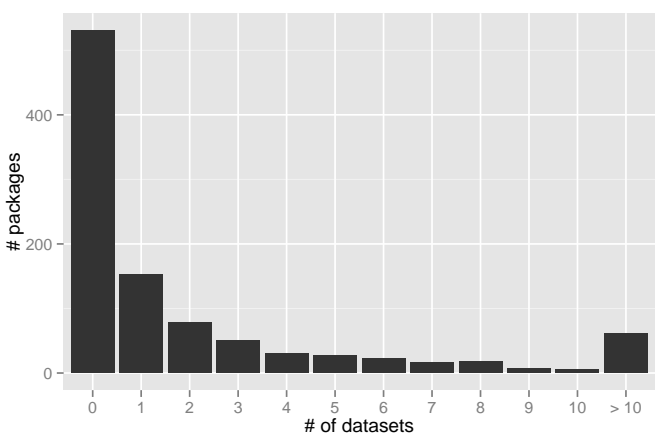

Figure 1: Basic summary of the number of datasets included in packages released on CRAN between Oct 5 and Nov 7 2012. The 1001 packages considered contain 3289 data objects in total.

Graphics help identify data quality issues; they reveal dataset structure and support the interpretation of results. The examples given here are taken from various packages and are more of a convenience sample than a random sample. In each case we give a brief description of the dataset, a reference to the dataset description in $\mathrm{R}$, and sometimes some additional relevant information. We offer a selection of graphics, each one pointing out some feature of the dataset, and the code to produce them. Given the power of R's graphical tools and how easy it is to draw graphics, there is no need to restrict ourselves to a single all-encompassing display for a dataset. Instead, we recommend drawing many graphics to ensure that most, if not all, aspects of the dataset are presented. This approach takes up a lot of space in a printed copy, but is easy to use on a computer screen or a webpage.

We would like to see more use made of graphics with $\mathrm{R}$, though only if each additional graphic conveys additional information. There is no value in drawing more graphics just because we can; they all have to tell a story. For each of the graphics in this article we have attempted to explain what particular information can be gathered from it. Sometimes there is overlap, in that the same information may be visible in more than one graphic. Also there may be alternative graphics readers might prefer to have drawn for presenting the same information. Graphics will always be very much a matter of taste and experience. As long as we are prepared to consider many graphics, we should be able to find good ones, which convey the information we want to draw attention to effectively.

Of course, graphics alone are not enough, just as analysis alone is rarely enough. We do not discuss any modelling here, as we concentrate on what graphics can contribute. Occasionally, we do point 
out where modelling would be especially useful in checking or confirming an idea discovered from a graphic. In many cases a suitable model is obvious; in some others it is uncertain if there is any model that would do. Graphical displays are able to draw our attention to unusual features in a dataset, which we can recognise as being interesting without being clear how to test for them.

\section{Examples}

\section{Anorexia - MASS}

This dataset is used in the MASS package (Venables and Ripley, 2002) to illustrate a linear model fit, using glm. No plot is given with the example code. The data is also used in many other packages such as granova (Pruzek and Helmreich, 2010) where some complex plots are provided. The original source is Hand et al. (1993) who recommend "whichever statistical technique is employed, it is instructive to look at the three scatterplots of after/before". Curiously, none of the R users of the data follow this advice.

The dataset contains pre- and post-treatment weights for 72 young girls being treated for anorexia. Three treatment groups are considered: cognitive behavioural therapy (CBT), family therapy (FT) and a control group (Cont). We suggest adding code to the help page for the anorexia dataset (?anorexia) starting with:

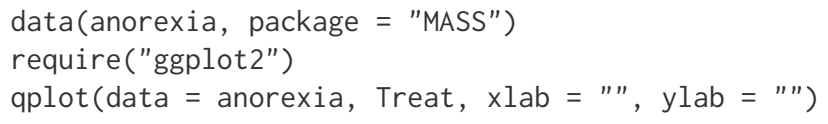

This results in a barchart of the numbers in the three groups shown in Figure 2. This plot, as all of our plots, is drawn with the packages ggplot2 (Wickham, 2009) and vcd (Meyer et al., 2006) and weaved into the document with knitr (Xie, 2012).

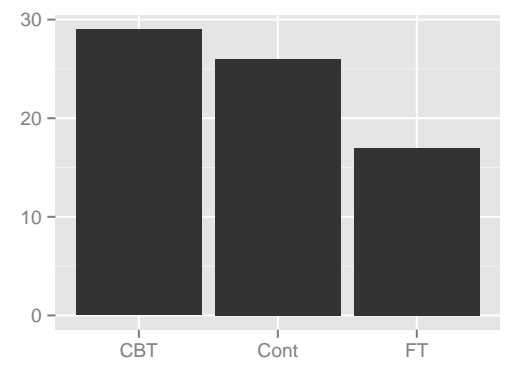

Figure 2: Barchart of the numbers of girls in each group in the anorexia study.

Story of Figure 2: This plot shows 'just' data structure, but surprisingly the three groups are not of equal size. This may mean that some girls dropped out or that it was an observational study without random allocation of the participants, or there could be another explanation altogether. This should be borne in mind in interpreting the results.

Figure 3 shows - as originally suggested by Hand et al. (1993) - scatterplots of the weights of the girls in the three treatment groups. A simple version of this plot can be achieved with the code below:

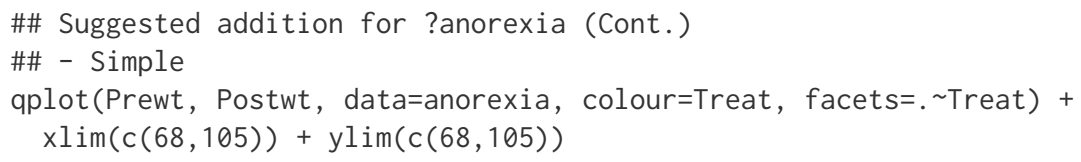

A more polished variation also includes guide lines and leads the following code and Figure 3.

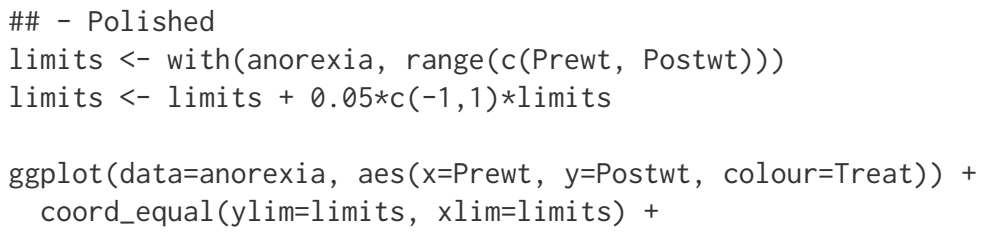




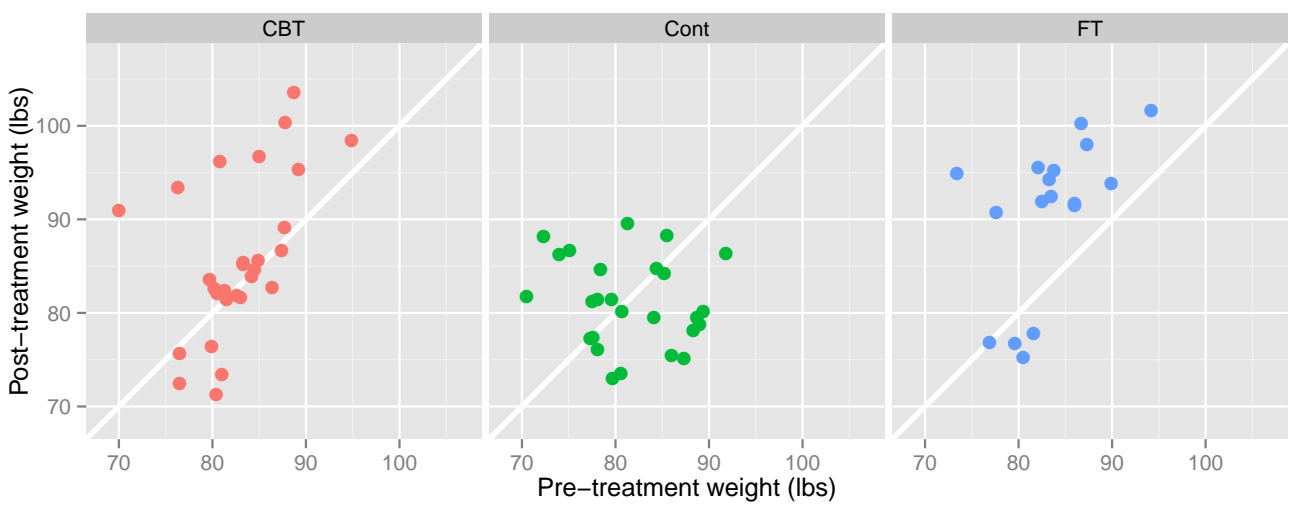

Figure 3: Scatterplots of pre- and post-treatment weights for the girls being treated for anorexia. $x=y$ guide lines have been added to help identify girls who gained or lost weight.

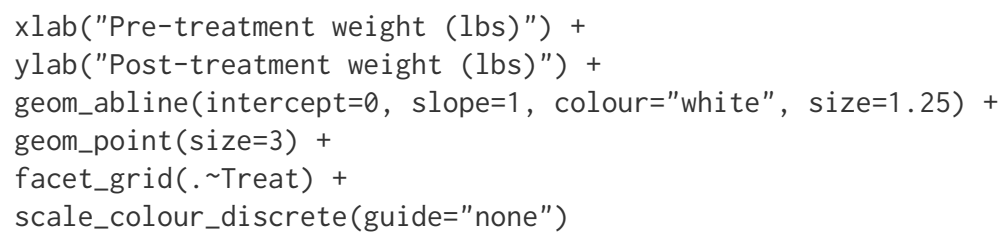

Story of Figure 3: This plot gives a preliminary overview of the data. We learn that there were differences in the weight gains between the treatment groups. Girls in the control group tended to have lower post-weight, and roughly half lost weight. Girls in the family treatment group fall into two clusters: girls in one group tended to respond well to this treatment and gained weight, but for a small group of four girls this treatment was not successful. Similarly, there appear to be two groups of girls in the CBT group: a group of about eight girls responded well to the treatment, but a larger group of girls did not respond well.

A general linear model to fit post-treatment weight given pre-treatment weight is suggested in the existing help file of the function glm, as shown below. To this, we add graphical support as shown in Figure 4, which displays plots that support a linear model with change in weight plotted against pre-treatment weight. A guideline is drawn at zero. Noticeable differences can be seen between the treatments. This results in the following overall code:

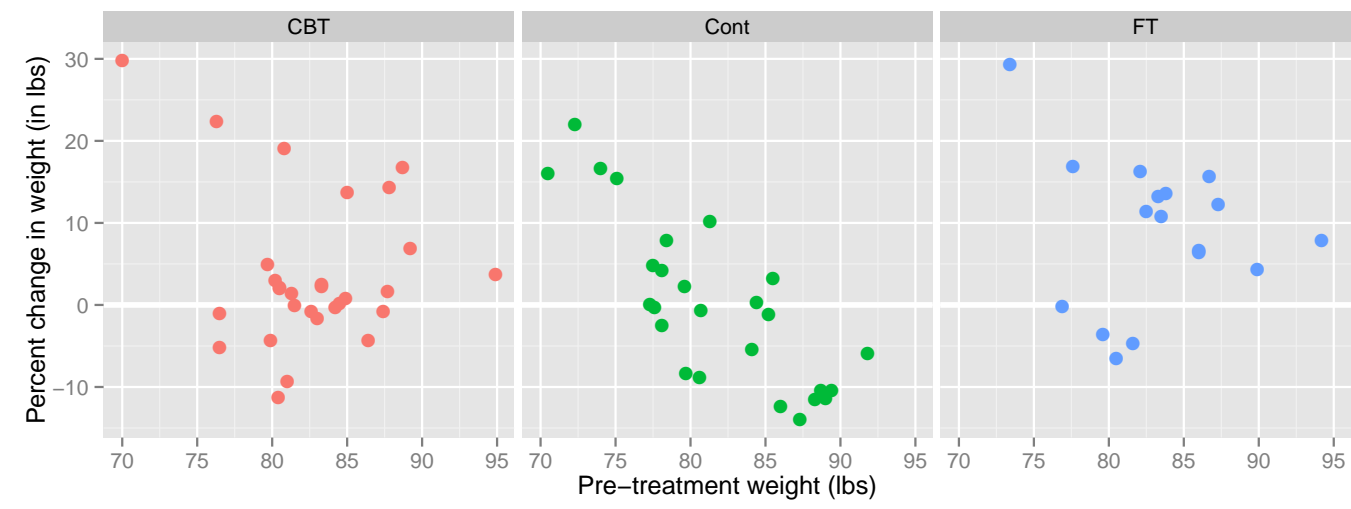

Figure 4: Percent change in weight by pre-treatment weight.

\#\# Existing example code

?glm

anorex. $1<-$ glm(Postwt Prewt+Treat+offset(Prewt), family = gaussian, data = anorexia)

summary (anorex.1) 


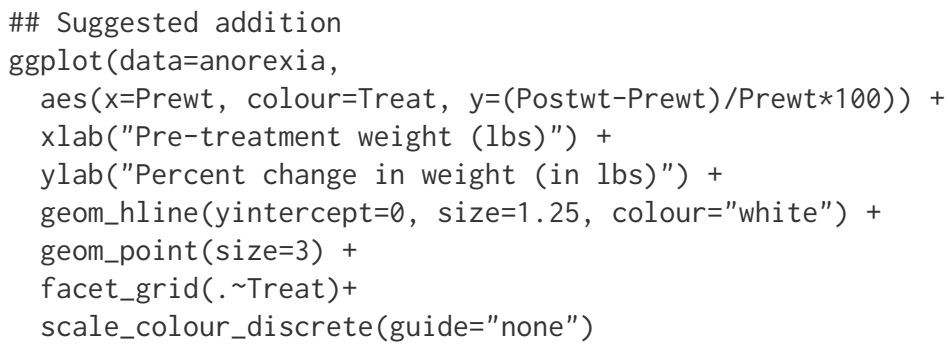

Story of Figure 4: There are differences in the weight gain between the treatments, but there is also a lot of individual variation. For some girls the two treatment methods clearly did not help them to gain weight. More girls in the control group failed to gain weight - only the very low weight girls gained much weight, which suggests that other influences may have been at work for these girls. The original data description indicates that intervention methods were used for the lightest girls. Generally there appears to be an association that the heavier girls, all still under normal weight, gained less weight than the lighter girls.

Various decisions were made in producing these plots to make them more informative.

- A raw data overview plot shows the data "straight out of the box". Such plots help the reader to identify any major issues with the data, such as extreme values or clusters.

- Colour was used to draw attention to the primary comparison of distributions of treatments. Facetting was chosen over plotting on the same plot because of the considerable variation within treatments, which makes it difficult to evaluate differences. Note that facetting, lattice structures (Sarkar, 2008), or trellis plots (Becker et al., 1996) all describe the process of positioning plots of different, usually mutually exclusive, subsets of the data next to each other. The subsetting is usually done by conditioning on co-variates. Conditioning has a central role in statistical data analysis, and finds its visual expression here.

- Guides for assisted viewing may be added, in this case the $\mathrm{x}=\mathrm{y}$ line to determine which girls gained or lost weight. In other situations we could also employ data driven guides, such as contours, to focus attention on patterns.

- The restructured data plot shows the data as a support for the model. Although weight change is not the precise match to the response variable, the model is implicitly exploring this. It also allows reading of the weight change in a vertical direction instead of the off-diagonal view in the raw data plot. The horizontal guide line at zero allows the reader to see which girls gained weight. Using a scatterplot enables the reader to see the nuances of the data, such as clusters of girls who do well in one treatment, or fail to respond, and the indication that intervention may have been applied to the very underweight girls.

\section{Lanza - HSAUR2}

The Lanza data (Everitt and Hothorn, 2006; Lanza, 1987) consists of four clinical studies on the use of the drug Misoprostol to prevent gastrointestinal damage. Damage was measured as an ordinal response classification with levels 1 to 5 corresponding to a specified number of haemorrhages (with 1 being the fewest and 5 the most). The visualisation given in ?Lanza is a set of four mosaicplots (without obvious information on which study is shown) shaded by residuals for an independence model.

Rather than a modelling result, we suggest the use of barcharts as a first introduction to the data. A simple barchart of study by treatment (not shown here) shows that all studies were balanced and that studies I to III had comparable numbers of patients, whereas study IV was smaller with just less than half the number of participants of the other studies. Breaking down the data further by the damage scores gives the multiple barcharts shown in Figure 5, requiring a single line extension of the existing code as shown below. We are making use of the small multiples paradigm (Tufte, 1983; Becker et al., 1996) to show study outcome in terms of number of haemorrhages in subsets by clinical trial and treatment:

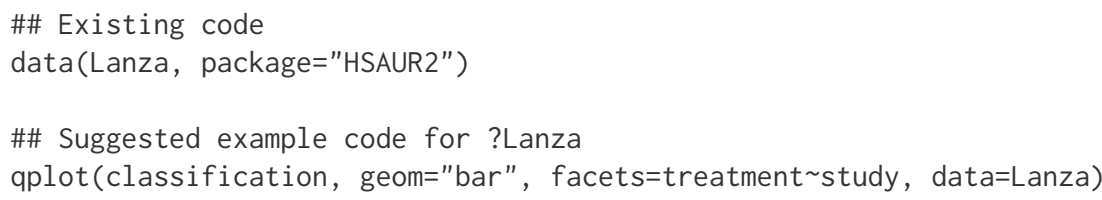




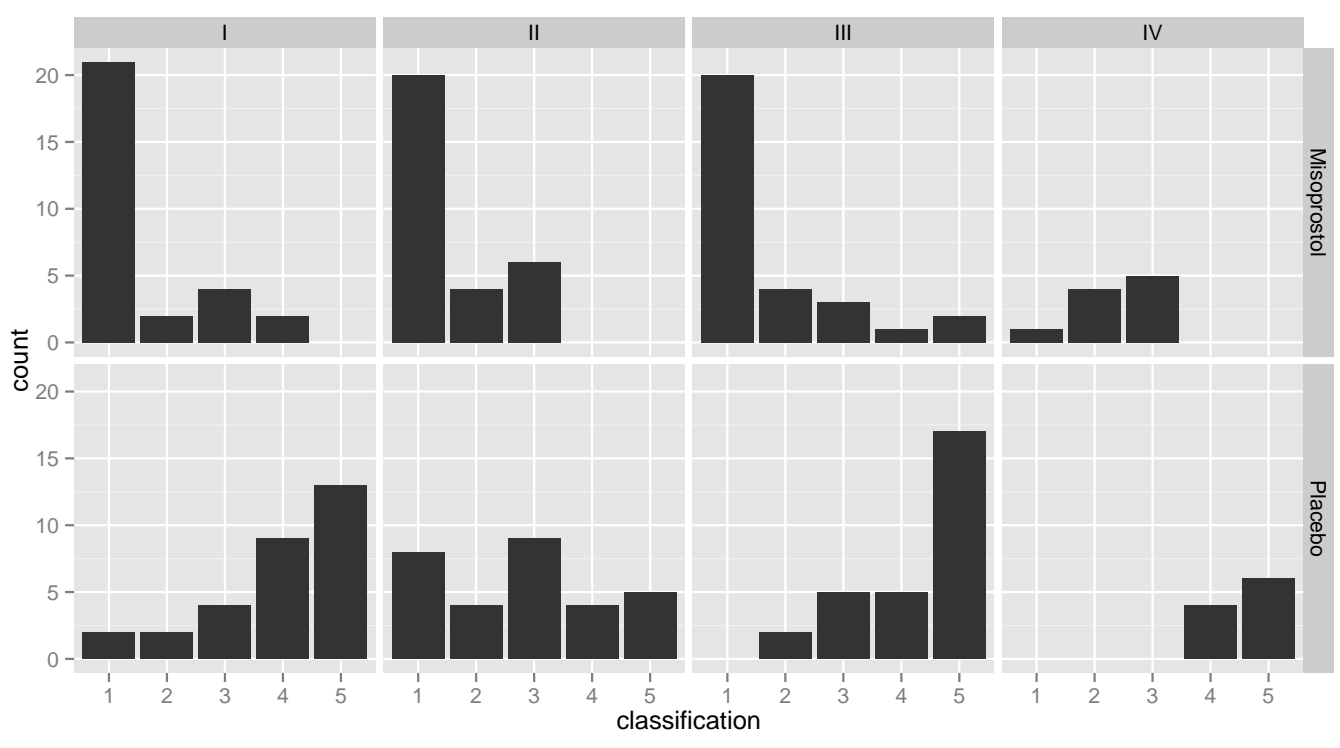

Figure 5: Number of haemorrhages classified from 1 (= best) to 5 (= worst) for patients in four clinical trials (left to right) treated with Misopropol (top row) or Placebo (bottom row).

Story of Figure 5: All of the barcharts suggest that Misopropol is an effective drug for controlling or preventing haemorrhaging - in every study patients in the active treatment group reported better end results than the Placebo group. The distribution of these results was very similar for studies I and III, with the bulk of people in the Misopropol group being classified as ' 1 ', and the bulk of patients in the Placebo group classified as ' 5 '. Study II showed a larger spread and less structure in the results for the Placebo group, while study IV showed the strongest distinction between results for the two treatment groups. Patients in treatment group IV also showed a different pattern of resulting haemorrhaging. Even in the active treatment group, the most frequent response was ' 3 ' - which might indicate, together with the relatively low number of patients, that study IV was done for a different target population with possibly more affected individuals.

Figure 6 is closer to the modelling approach suggested by the existing help file. The code for showing this figure is:

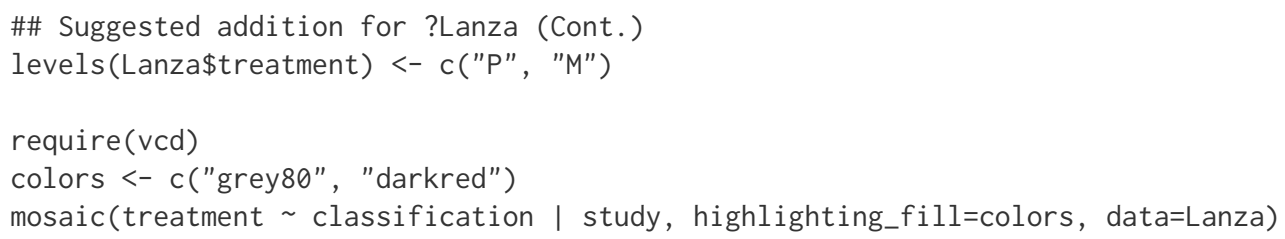

\begin{abstract}
Story of Figure 6: A mosaic shows estimates of the conditional probability of being in the active treatment group given a particular haemorrhaging classification was reported. Since we know from our initial overview that treatment groups were balanced, this conditional probability is proportional to the conditional probability of seeing a specific outcome given treatment group, which is what we are more interested in, given the setup of the study. Again, we see that all four studies suggest a high effectiveness of Misopropol in terms of preventing haemorrhaging, since the presence of patients treated with Misopropol decreases with an increase of haemorrhages. Again, the different number of patients in study IV is visible (from the lower height of the rectangles in the last row of the plot), as well as its stronger separation of haemorrhaging outcomes between treatment groups.
\end{abstract}

\title{
schizophrenia2 - HSAUR2
}

The schizophrenia2 data are from a ten-month longitudinal study following 44 individuals suffering from episodes of schizophrenia. Every two months (except at 4 months after the study began) 


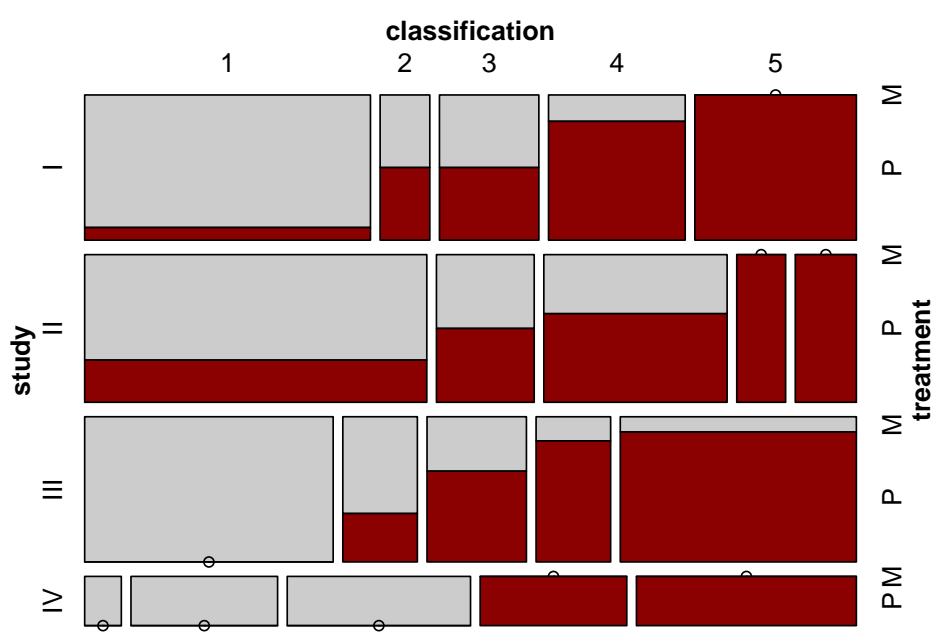

Figure 6: Mosaicplot of the Lanza Data: each row corresponds to one of the four clinical trials. Classification of haemorrhaging is shown from left to right. Patients in the Misopropol group (active treatment) are shown in red, patients in the Placebo group are shown in grey.

individuals reported whether the disorder was in an active state (present) or dormant (absent). Additionally, the data contain information about age at onset of the disease: under the age of 20 or, less often, over the age of 20.

The current help file presents a mosaicplot as an overview of the data, which we basically agree with, albeit we would like to suggest changes to the default values to emphasise various features in the data:

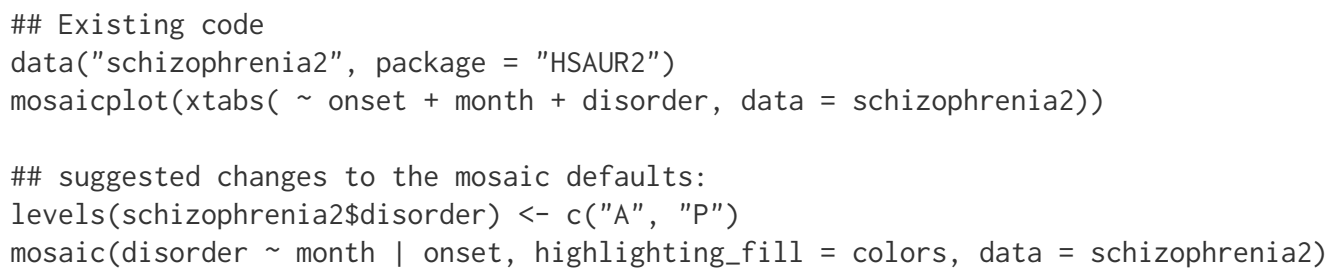

Changes to the defaults are based on recognition principles: reducing the spacing between rectangles emphasises grouping of bins and thereby helps with cognition of comparable subsets (Ware, 2004). Similarly, the pre-attentive property of colour (Healey, 2009) is used to give a strong additional visual cue of similarity. The change from vertical to horizontal layout emphasizes the longitudinal aspect of the study; by making the time component 'month' the $x$ axis, we adhere to the convention of time-series plot.

Story of Figure 7: This plot gives an overview of the schizophrenia2 data. For both early onset (top row) and late onset of the disease (bottom row) the state of the disease is shown for every two months after the beginning of the study - with the inexplicable exception of 4 months after the study began. Individuals reporting the disease are coloured dark red, the remaining cases are shown in grey. As the study progressed, fewer individuals experienced episodes of schizophrenia. The slight shifts in vertical alignments of rectangles between onset classifications (upper and lower row of the mosaic) hint at the presence of missing values.

The next plot incorporates these missing values, but leaves everything else unchanged. The code for that is given as:

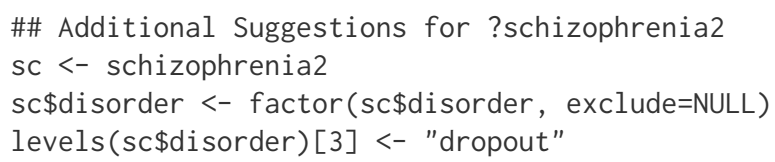




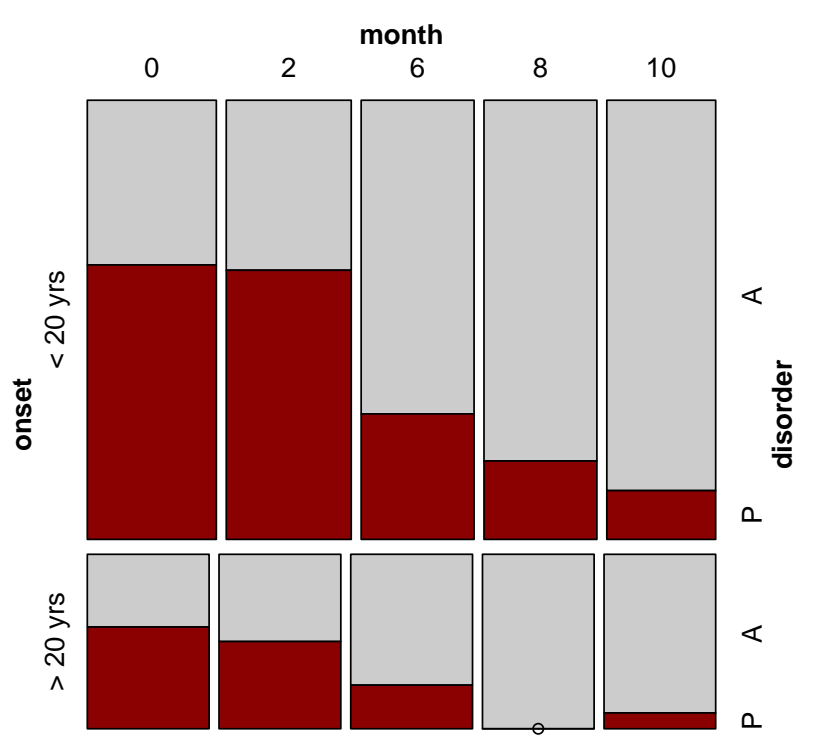

Figure 7: Mosaicplot of the schizophrenia2 Data: 44 female patients were tracked over 10 months (from left to right) after hospitalisation, their disorder status is recorded as absent or present (grey versus dark red). Top and bottom row of the mosaic correspond to early onset of the disease (top row) versus onset after 20 years of age (bottom row).

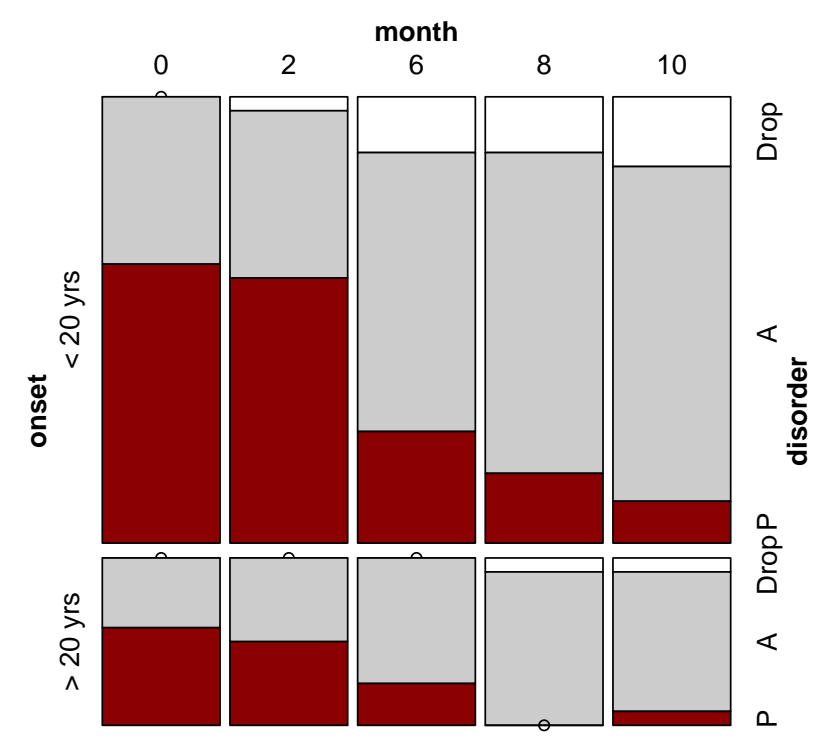

Figure 8: Mosaicplot of the schizophrenia2 Data: the setup of the plot is the same as in Figure 7, but drop-out patients are now emphasised (white rectangles). Drop-out rates increased as the study progressed. Note that rectangles between different onset times of the disease are now aligned vertically. 
sc\$disorder <- factor (sc\$disorder, levels=rev(c("present", "absent", "dropout")))

colors <- c("white", "grey80", "darkred")

mosaic(disorder month | onset, highlighting_fill = colors, data = sc)

Story of Figure 8: Figure 8 emphasizes the presence of drop-outs from the study: the number of drop-outs increased as the study progressed. Drop-out rates between different onsets of the disease can be made comparable by changing the plot to a Doubledecker display using the parameter direction=c ("v", "v", "h") in the code above (not shown).

Figure 9 summarizes individuals' experience throughout the study. Again, a mosaicplot is our suggested visualization. Ordering of the individuals is crucial for the plot and requires a surprising amount of coding to achieve the display we want. Sorting is an important, yet underestimated and underused tool in statistical graphics.

The following code chunk leads through all necessary steps necessary for producing Figure 9:

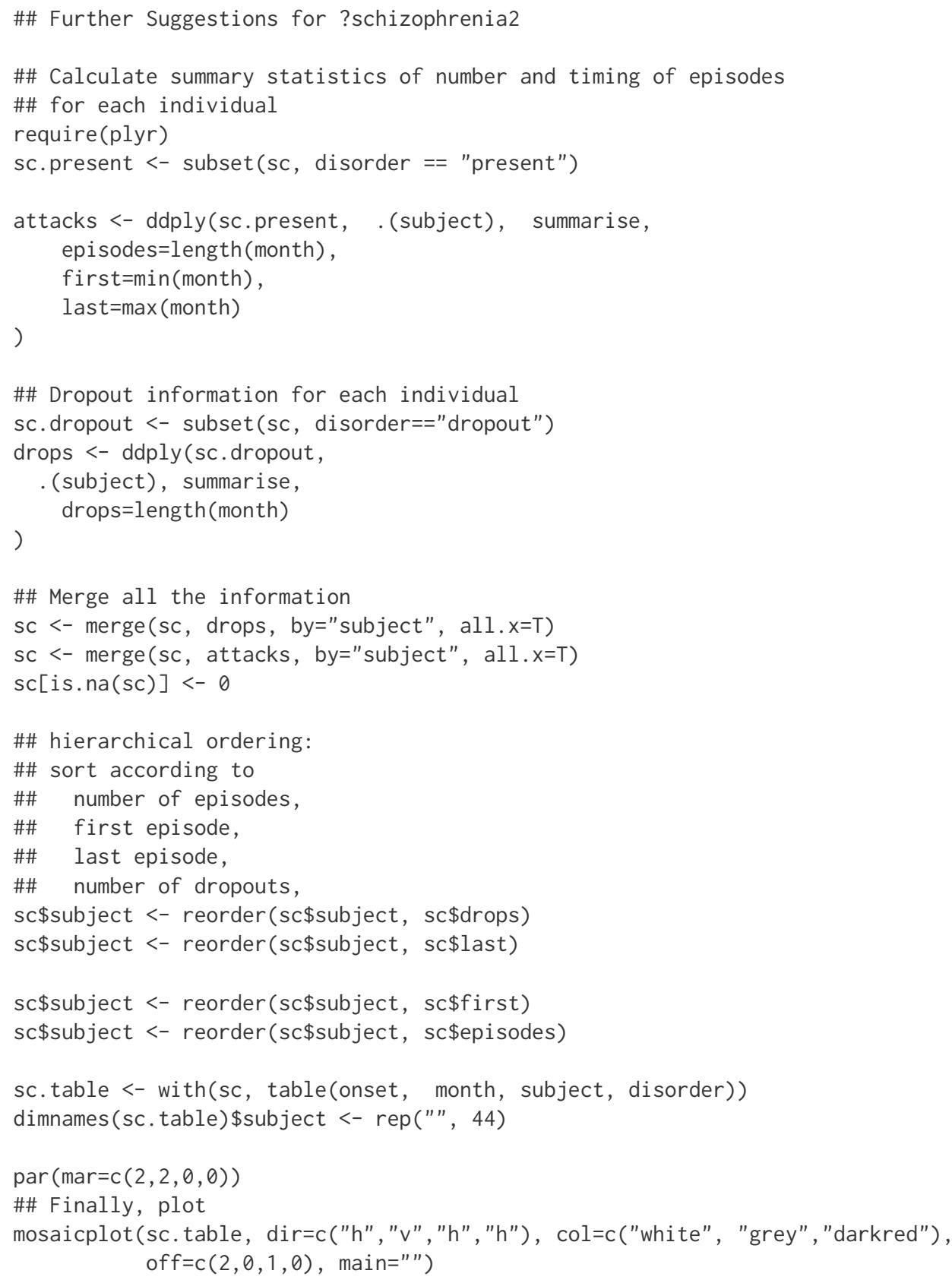

Story of Figure 9: The mosaic plot of Figure 9 shows each scheduled reporting of disease status for each individual in a box coloured according to the disease status (red for present, 

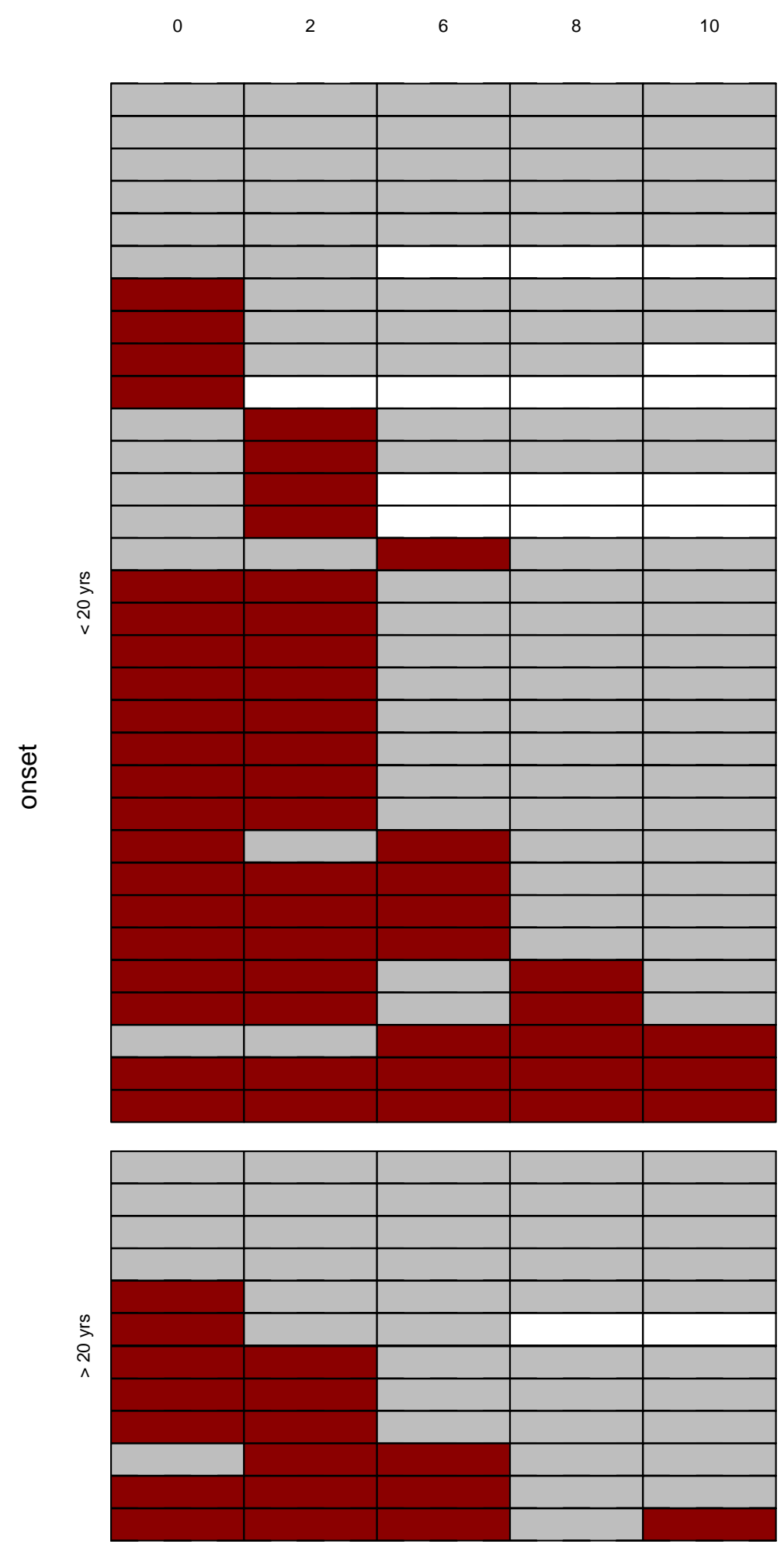

month

Figure 9: Mosaicplot of the schizophrenia2 data focussing on individuals' experience throughout the study. Individuals are ordered according to the numbers of times absence or presence of the disease were reported. 
grey for absent, white for not reported). Time in months is on the horizontal axis and each individual is represented in a horizontal row of boxes. In this setting, we distinguish early onset (top rows) versus late onset of the disease. Within each of these groups, subjects are ordered hierarchically according to number of episodes reported, first reported episode, last reported episode and lastly, number of non-reports. What becomes apparent is a pattern in the non-reports: once a person does not report for the first time, consecutive reports are missing as well. Additionally we see that the majority of participants do not suffer episodes at 8 or 10 months, if they did not report an episode beforehand. The similarity of reports is also remarkable; ignoring non-reports, there are potentially $2^{5}=32$ different patterns of reports - only 12 of these patterns show up among the 44 participants.

\section{SexualFun - vcd}

The SexualFun dataset contains responses of 91 married couples to the questionnaire item "Sex is fun for me and my partner". Each individual reports on a scale of "Always fun", "Very Often", "Fairly Often", and "Never Fun". The only visualization in the current version of ?SexualFun is an agreement plot (Friendly, 2000; Meyer et al., 2010), which is based on Bangdiwala agreement statistics. As a precursor to that, we suggest the use of multiple barcharts, as shown in Figure 10, resulting from the code below, or a fluctuation diagram (Hofmann, 2000).
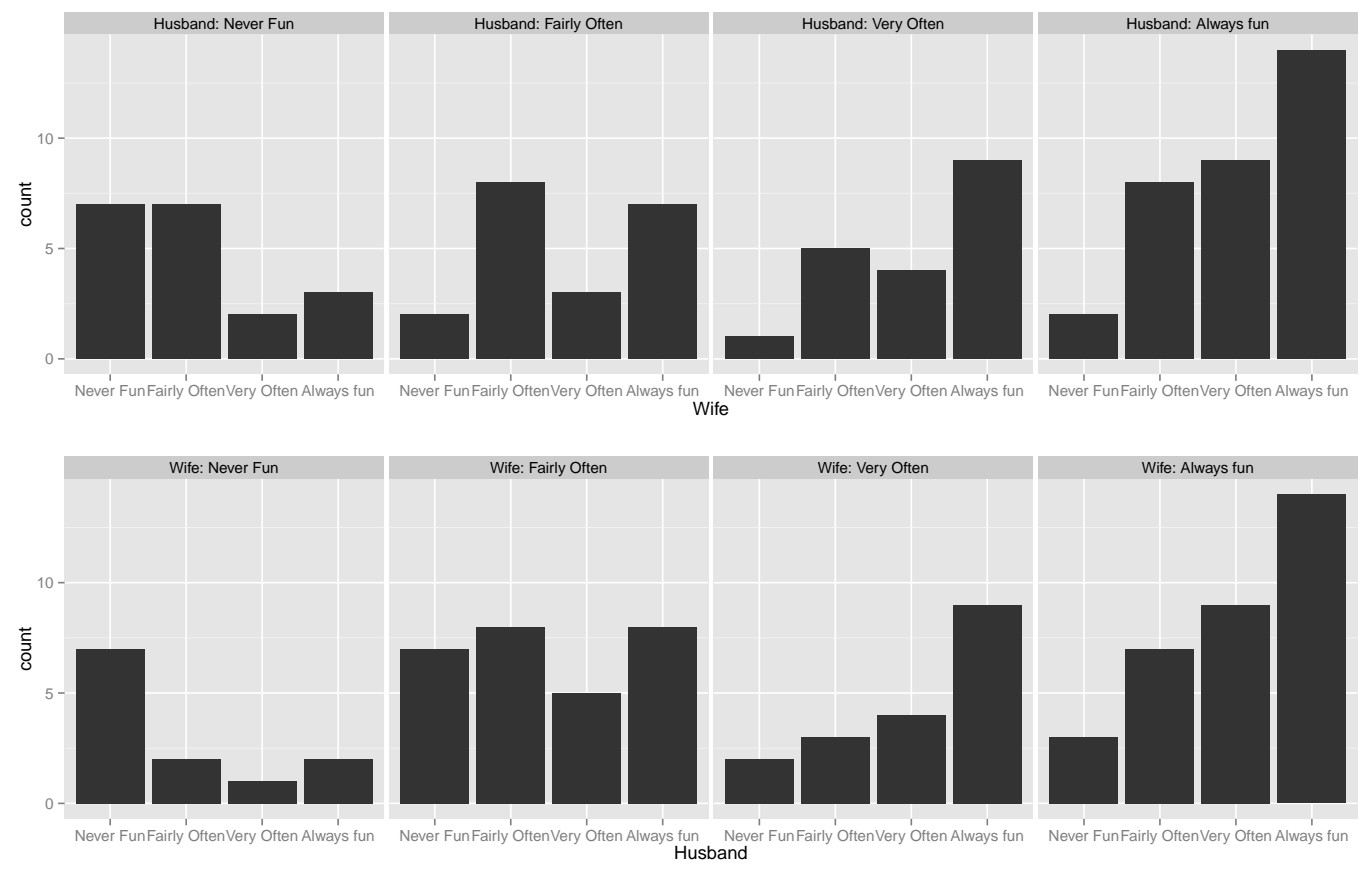

Figure 10: Barcharts of SexualFun showing the conditional distributions of responses to the questionnaire item "Sex is fun for me and my partner", conditioning first on the husband's responses, then on the wife's.

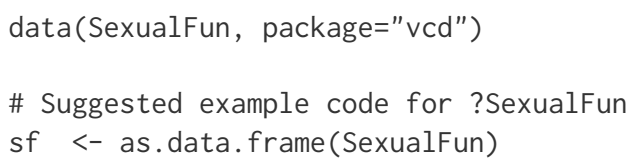

qplot(Wife, data=sf, weight=Freq) + facet_grid(facets=. Husband, labeller="label_both") qplot(Husband, data=sf, weight=Freq) + facet_grid(facets=. Wife, labeller="label_both")

Story of Figure 10: The conditional barcharts show that the distributions are very similar except for the combinations "Never Fun" and "Fairly Often", where the wives say "Never Fun" less frequently. Frequencies of "Always fun" are approximately equally high for both husbands and wives. In general, responses are spread across all possible combinations, with larger differences between couples occurring less frequently. 
Assuming integer scores 1 to 4 for levels "Never Fun" to "Always fun", we can compute a joint score for each of the couples as the sum of their answers and an index of disagreement as the difference between husbands' and wives' scores. Plotting this is similar to a Tukey transformation (Tukey, 1977), i.e. a rotation of a fluctuation diagram by 45 degrees.

This is achieved by the code below. Note that the command ggfluctuation is deprecated in the current version of ggplot2, but still works locally (after removing the deprecation notice).

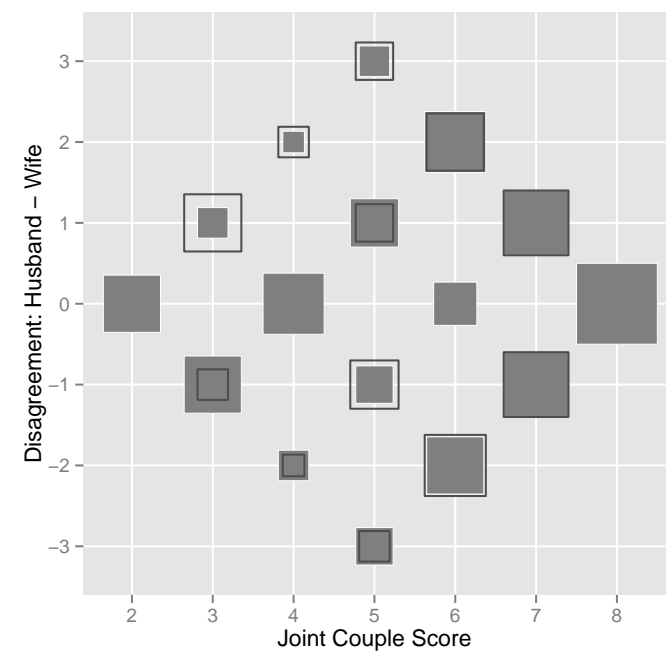

Figure 11: Rotated Fluctuation Diagram of SexualFun: disagreement of the partners is plotted on the $y$ axis against the joint couple score on the $x$ axis. Black rectangles are overlaid - these show the mirror image along the horizontal axis to emphasize differences in husbands' and wives' scores.

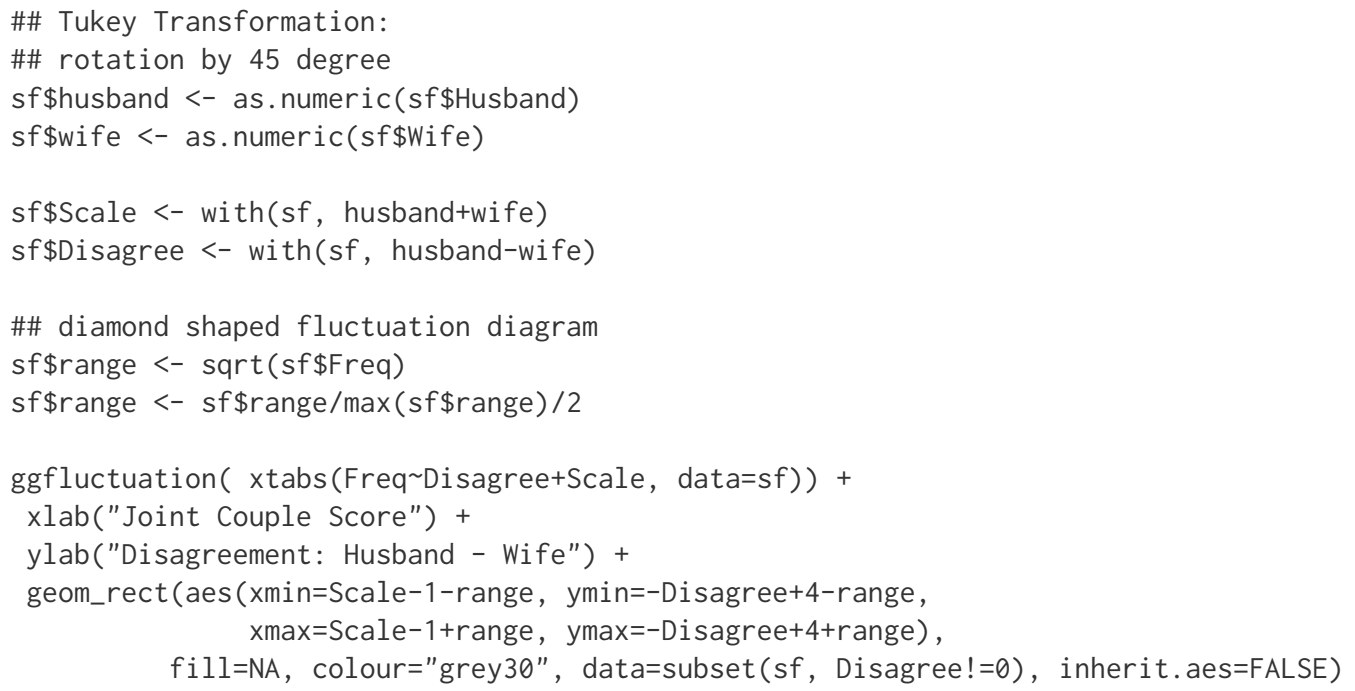

Story of Figure 11: This rotated fluctuation diagram emphasizes the numbers of agreements and disagreements among the couples directly. Symmetry above the middle line and asymmetry below reveal agreement and disagreement between husbands and wives. The fluctuation diagram is overlaid by outlines of the other spouses' squares to highlight deviation from symmetry. The top left corner again confirms what we have already observed: fewer women than men responded with "Never fun".

Figures 10 and 11 show the same information, though in Figure 10 each data point is shown twice. Figure 11 is more efficient but requires more work to decipher. With the overlaid rectangles it is in particular helpful to highlight asymmetry in the answers between husbands and wives. There are many more alternatives to the plots that we have chosen. One alternative to the set of barcharts would be stacked barcharts, for instance the likert plots implemented in Richard Heiberger's HH package (Heiberger, 2012). Interactive graphics would be beneficial to both. 


\section{Discussion and conclusions}

What do we want? We would like to ask $\mathrm{R}$ Core to add a recommendation in the existing test suite for packages to show plots within the example section of a dataset documentation. We don't ask for this lightly, and it certainly is not an ideal situation, since it adds yet another hurdle that developers need to pass on their way to creating and submitting a package on CRAN - nor does it guarantee that the plots suggested would be very helpful. However, this falls in line with standard rules for CRAN - checks are not based on content, but are syntactical. This is done in the hope, that syntactic violations, for which we can create automatic tests, are correlated with offenses in terms of content. From a technical point of view the infrastructure of the package system is already there. A plotting recommendation for datasets is feasible without introducing a massive overhead because of dependencies on various graphics packages: the Suggests specification in the description file takes care of exactly those situations. It allows the listing of all packages that do not need to be loaded for a package, but are necessary for successfully running the examples.

Data is at the heart of our discipline. Having stricter rules on data descriptions will give data a more central position in the $\mathrm{R}$ community. The absence of good data documentations, both in terms of the data structure and potential findings, devalues its usefulness. Documentation is a large part of achieving reproducibility of research results, which has been one of R's goals (Gentleman and Temple Lang, May 2004) for a long time. Past (Leisch, 2002) and recent advances (Xie, 2012) in tools for reproducible research have facilitated the use of the existing infrastructure so much that we should more actively employ it.

Help files do not currently include any output, neither graphics nor any model output, and that is probably a good thing. Users can simply run the code and see for themselves what happens. What we are asking for are some pieces of code in the help files for creating (sensible) graphics along the lines shown in this paper.

All our examples share the same unifying themes. Simple initial graphics give a good overview of basic information - like the sizes of treatment groups and potential differences between them and identify problems with the data — such as data errors, outliers or heaping. Graphics can enhance data analyses by suggesting new angles and by illustrating results. Graphics have to tell a story just as any statistical model must and stories should be given explicitly in the text to the graphics. Stories in graphics are rarely as "obvious" or "easy to see" as is often claimed. From a plot creator's point of view this gives us also a way to check if a plot is worth a story — if we are having a hard time telling the story, we might want to take this as a hint, that we should change the plot to something that is easier to talk about.

Presenting a collection of graphics is usually better than trying to convey all of a dataset's information in a single display.

\section{Bibliography}

R. A. Becker, W. S. Cleveland, and M. J. Shyu. The visual design and control of trellis display. Journal of Computational and Graphical Statistics, 5(2):123-155, 1996. [p120]

B. S. Everitt and T. Hothorn. HSAUR2: A Handbook of Statistical Analyses Using R. Chapman \& Hall/CRC, 2nd edition, 2006. URL http://CRAN.R-project.org/package=HSAUR2. R package version 1.0-2. [p120]

M. Friendly. Visualizing Categorical Data. SAS Publishing, 2000. [p126]

R. Gentleman and D. Temple Lang. Statistical analyses and reproducible research. Bioconductor Project Working Papers, May 2004. [p128]

D. J. Hand, F. Daly, K. McConway, D. Lunn, and E. Ostrowski. A handbook of small datasets. Chapman \& Hall/CRC, 1993. [p118]

C. Healey. Perception in visualisation, 2009. URL http://www.csc.ncsu. edu/faculty/healey/PP/ index.html. [p122]

R. M. Heiberger. HH: Statistical Analysis and Data Display: Heiberger and Holland, 2012. URL http: //CRAN. R-project. org/package=HH. R package version 2.3-17. [p127]

H. Hofmann. Exploring categorical data: Interactive mosaic plots. Metrika, 51(1):11-26, 2000. [p126]

F. Lanza. A double-blind study of prophylactic effect of misoprostol on lesions of gastric and duodenal mucosa induced by oral administration of tolmetin in healthy subjects. British Journal of Clinical Practice, pages 91-101, 1987. [p120] 
F. Leisch. Dynamic generation of statistical reports using literate data analysis, 2002. [p128]

D. Meyer, AchimZeileis, and K. Hornik. The strucplot framework: Visualizing multi-way contingency tables with vcd. Journal of Statistical Software, 17(3):1-48, 2006. URL http: / /www. jstatsoft. org/ v17/i03/. [p118]

D. Meyer, A. Zeileis, and K. Hornik. vcd: Visualizing categorical data., 2010. [p126]

R. M. Pruzek and J. E. Helmreich. granova: Graphical Analysis of Variance, 2010. URL http: //CRAN. Rproject. org/package=granova. R package version 2.0. [p118]

D. Sarkar. Lattice: Multivariate Data Visualization with R. Springer, New York, 2008. URL http: //Imdvr.r-forge.r-project.org. ISBN 978-0-387-75968-5. [p120]

G. K. Smyth. Australasian Data and Story Library (OzDASL), 2011. URL http: //www. statsci . org/ data. [p117]

E. Tufte. The Visual Display of Quantitative Information. Graphics Press, 1983. [p120]

J. W. Tukey. Exploratory Data Analysis. Addison-Wesley, 1977. [p127]

W. N. Venables and B. D. Ripley. Modern Applied Statistics with S. Springer, New York, fourth edition, 2002. URL http://www. stats.ox.ac.uk/pub/MASS4. ISBN 0-387-95457-0. [p118]

P. Vlachos and M. Meyer. StatLib: Data, Software and News from the Statistics Community, 1989 -. URL http://lib.stat.cmu.edu/. [p117]

C. Ware. Information Visualization. Morgan Kaufmann, San Francisco, CA, USA, 2nd edition, 2004. [p122]

H. Wickham. ggplot2: elegant graphics for data analysis. Springer New York, 2009. ISBN 978-0-387-98140-6. URL http://had.co.nz/ggplot2/book. [p118,199]

Y. Xie. knitr: A general-purpose package for dynamic report generation in R, 2012. URL http://CRAN. Rproject. org/package=knitr. R package version 0.5. [p118, 128]

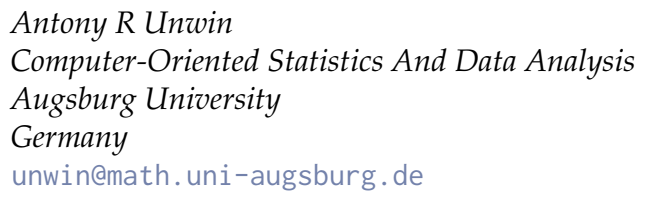

Heike Hofmann

Department of Statistics

Iowa State University

United States

hofmann@mail. iastate.edu

Dianne H Cook

Department of Statistics

Iowa State University

United States

dicook@mail.iastate.edu 